\title{
LOAD MONITORING SOLUTIONS FOR THE SMART BUILDINGS - AN OVERVIEW
}

\author{
F.C. ARGATU, VIOLETA ARGATU, B.A. ENACHE, C. CEPISCA, G.C. SERITAN, P.C. ANDREI \\ University POLITEHNICA of Bucharest, Splaiul Independentei nr. 313, Bucharest, Romania \\ E-mail: florin.argatu@upb.ro
}

\begin{abstract}
The development of intelligent smart grids requires new electrical monitoring solutions in order to optimize power consumption. The implementation of intelligent energy-monitors leads to new technical developments to optimize consumption using intrusive and non-intrusive techniques. This paper makes a review of the main methods used for load monitoring systems, indicating their advantages and disadvantages. The emergence of high-harmonic consumer electronics in electrical networks such as LED lighting sources implies the choice of the appropriate method and equipment for optimizing energy consumption based on energy signatures.
\end{abstract}

Keywords: load monitoring, intrusive methods, non-intrusive methods, load signature

\section{INTRODUCTION}

For monitoring and controlling electrical energy consumption in smart buildings, home and residential places, is very important to know the load signature for individual consumers. The load signature is represented by the set of measurable electrical parameters which can be detected by the installed measurement apparatus at a given time. Besides monitoring it is also essential for the estimation of energy consumption, to track the power quality of the energy supplied. To achieve all this there are several solutions for monitoring the electrical appliances.

The first solution involves the acquisition of the electrical parameters for different consumers at different operating levels by using a network of wireless sensors mounted inside the appliances (Intrusive Load Method ILM). In this case, each device of the electrical grid must be connected to a smart sensor that measures its consumption. The information thus obtained from the sensors is processed and sent to the central server generating a report [1], [2]. This solution involves different types of equipment and a high costs for acquisition and implementation. The advantage is represented by its accuracy and reliability of the measurements since only the errors of the measuring instruments are presented in the final results of energy measurement.

Another solution refers to the use of a single intelligent monitoring device (Non-Intrusive Load Method - NILM), most frequently installed at the entrance of the home's electrical network. Based on the load signature determined for each consumer, it is possible to determine which of them are turned on. By reducing the number of sensors and equipment needed for monitoring all consumers, the costs are minimized, implicitly reducing the complexity of the network [3], [4].

So far, from a practical point of view, there is an early development of monitoring equipment based on NILM. Simultaneously with the development of "smart home" systems, their users are increasingly expecting optimization of energy consumption, safety, and intelligent services [5]-[7].

The researchers aim, and motivation is to offer users the prospect of saving energy and money in the simplest way possible, preventing possible electrical malfunctions and recognizing the activity of the electrical installation components based on data recorded over a specified period. Another direction for researchers is to determine the aspects related to the quality of the electricity and to optimize the different types of consumers using their load signature.

This paper presents actual directions to follow for researches and methods and equipment designated for load monitoring for consumers.

\section{NILM SOLUTIONS FOR THE LOAD SIGNATURE}

\subsection{System structure}

The process of obtaining a consumer's load signature through NILM consists of two main steps: data acquisition and data processing (Figure 1).

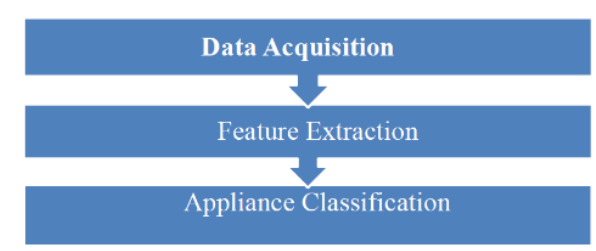

Figure 1. A general framework for NILM approach

Generally, the hardware for the acquisition system has two transducers one for current and one for voltage. The power factor is calculated based on these values for the active power, the reactive power, and the apparent power. There are also other advanced measurements, such as measuring the harmonic distortion [8], [9], transient magnitudes, electromagnetic interferences all of these measurements leading to the achievement of NILM's primary objective, namely to estimate the operating status of individual consumers and their energy consumption. 
The factors that affect NILM performance are a range of power measurement, sampling rate, A/D conversion resolution, etc. The NILM system has evolved over the years through improvements for its hardware features (such as higher sample sampling rates to manage such transitions), and improved algorithms to recognize a variety of appliances in residential buildings as well commercial buildings. Appliance feature extraction module, inference and identification module and learning module are the essential parts of NILM system structure.

Depending on the data acquired on the sampling rate are two broad sets of hardware used for NILM [10]-[12]. Equipment with low sampling rate $(1 \mathrm{~Hz})$ which is a standard and cheap equipment [13], and equipment with high sampling rate $(>5 \mathrm{kHz})$ requiring a complex structure and therefore high costs.

\subsection{Appliance types}

Depending on the structure and operating regime of the electrical appliance, they can consume different amounts of energy. The resistive loads use the power exactly as it comes from the source. The current being in phase with the voltage, the power is taking positive values and is being consumed entirely by the device. The inductive and capacitive loads consume the energy differently, due to the phase shift between the two components, power taking positive and negative values. In this case, only the active power, the positive value, is used by the device while the remaining power, reactive power, is stored and then returned to the source. The traditional electromagnetic equipment uses a sinusoidal current from a sinusoidal source; new equipment converts the alternating current into DC so a non-sinusoidal current appears, thus introducing new high frequencies, called harmonics. To be able to configure an electrical device detection algorithm, we need to know the types of electrical appliances.

"Permanent appliances devices" are those type of electrical devices that remain active through the entire day consuming energy at a continuous rate and therefore referred to as permanent [13], [14]. Appliances such as the hardwired smoke detector, telephone sets, cable TV receivers are amongst the devices belonging to this category [12].

The next category of appliances are on/off devices which change power consumption when switched on or off. For these devices the power changes when switching from the off state to the on state having the same value but opposite sign. By identifying combinations of power changes that satisfy these conditions, it is easy to identifying on/off consumers as well as estimating their consumption.

A problem may occur if many appliances change their state at the same time. As a solution, the Switch Continuity Principle was introduced in [15]. The sampling frequency of the acquisition system must be large enough to detect the power transitions of the equipment at short intervals. It is assumed that within a short time we have no power transitions. Thus, between two acquisition intervals, in which we have total fixed power, we can identify the equipment that changes its operating state [16].

Appliances with multiple states of operation are a special case of on/off type. After they are started they may perform several operations, each of which causes different power variations. In this category, we can find simple consumers or compounds. From the category of simple consumers belong these consumers who perform only one function, but have different energy levels. The typical example of this category is a fan with several gears it performs the same function, rotating the blades, but it can do it at different levels - rotation speeds. Compound consumers are those with multiple functionalities and can accommodate more simple consumers. An example would the washing machine, which can be seen as a combination between an electric machine drive, a water pump and possibly a heating component used for drying.

A standard way to represent this class of appliances is the finite state machine (FSM) model. Thus, the state of the finite automaton defines the state of operation for a device, while the transitions represent the passage for a consumer from one operating state to another.

The finite automaton states, the power level characteristic of the respective state and the transitions accompanied by a change in the power value are enough to represent the functionality of any consumer. Due to noise, variations in supply voltage or other causes, changes in power recorded at a consumer's transition from one state to another may take different values. This implies the need to consider a tolerance in the analysis of the power changes corresponding to each consumer. Figure 2 represents a finite state machine model with two states of operation, as illustrated. State A represents a power consumption of $500 \mathrm{~W}$ and state $\mathrm{B}$ a consumption level of $750 \mathrm{~W}$. At the transition from $\mathrm{A}$ to $\mathrm{B}$, the power consumption of the appliance rises with $250 \mathrm{~W}$. In contrast to this, the power consumption decreases by $250 \mathrm{~W}$ when the changes occur from B to A. This is analogous to Kirchhoff's law, as the sum of the total power changes is zero [16].

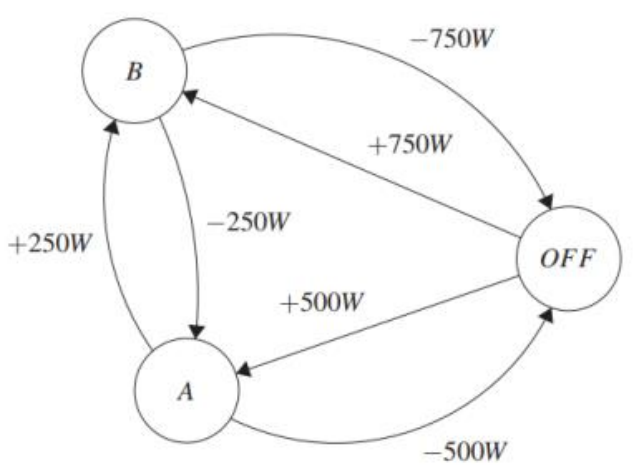

Figure 2. A finite state machine (FSM) for an electric heater [16]

Continuously variable electrical devices (CVD): the fourth class of electric device models are devices which 
have an infinite number of states. Continuously varying electrical devices such as light dimmers, sewing machines, and variable speed vibrators do not consume constant power and therefore do not generate clear power level change signals.

The energy consumption pattern of the different type of appliances has been shown in Figure 3, which is further interpreted as an appliance feature to discriminate between different appliance classes. New studies tend to focus on defining load signatures tailor-made to the appliance classes listed above to characterize them in a best possible way for identification. However recently in [10], an author has argued that appliances can have a multi-working model based on user customization and working styles; therefore this must be accounted into the feature extraction process [12].

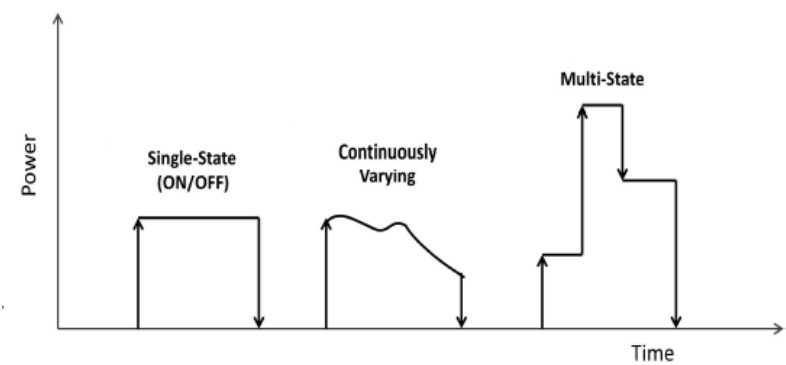

Figure 3. Different load classes based on their energy consumption pattern.

\section{THE ISSUE OF THE ELECTRICAL APPLIANCE SIGNATURE}

At the heart of every approach for monitoring and detecting, different appliances are the need to understand their electrical behaviour [17], [18]. NILM systems identify individual electrical devices based on their load signature [17], [19], and [20]-[22].

Electrical appliance signature can be defined as the behaviour of an electrical device during operation which is different for every device. Each electrical device contains unique features during operation similar to human footprint (hence the name of the electrical load signature of the device) [19]. Parameters that can be used to describe the electrical load signature include current, voltage, power measurements, harmonics, transient signal, on/off states, etc. Generally, for the identification of electrical appliances, NILM systems use two different types of load signature, steady-state and transient modes Figure 4, but recently there is a greater concern in obtaining consumer characteristics through so-called nontraditional methods.

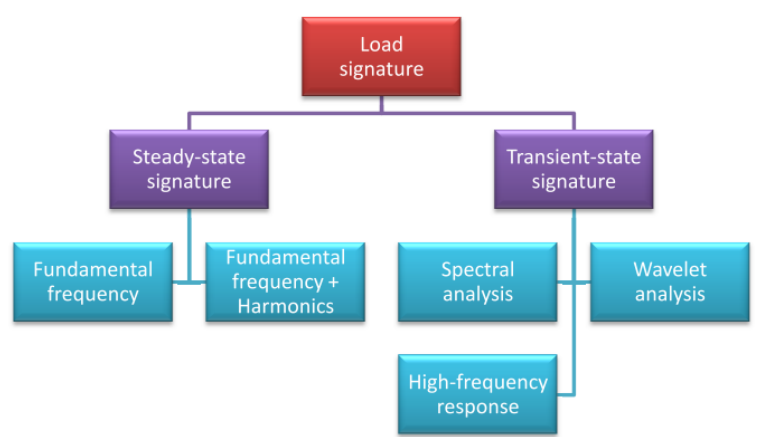

Figure 4. Classification of load signatures based on investigation methods [20]

\subsection{Load signature in steady state mode}

Steady-state analysis refers to the step changes in power signals (active power and reactive power) that occur when a particular electrical appliance stops or starts Figure 5 [15], [24].

Steady state analysis requires the use of changes that occur between two stable power states for the detection of electrical consumers. Over time, these changes have been used by various researchers to implement algorithms that allow non-intrusive detection of consumers [2], [14], [18], [25], [26], [27], [28], and [29]. Initially, G. W. Hart implemented a clustering system for consumer detection with two states: On/Off [15]. The values of active and reactive power during changes are determined and then analyzed for identifying variations that can be associated with passing a consumer from one state to another. In addition, permanently operating devices for continuous consumption cannot be detected by this method; the devices remain active 24 hours a day, 7 days a week, with constant active and reactive power values. Also, electrical devices that consume similar power, a computer and incandescent bulb, cannot be separated by this method [24] and [30]. The matching algorithm of this method is based on the assumption that the positive power shift (switch on) matches the negative power change (deactivation). However, the impedance load of many electrical devices changes over time, resulting in changes in energy consumption; a mismatch may arise due to this power deviation.

In [32] and [33] the authors proposed a different method using the V-I trajectory to classify a set of appliances. For each appliance, the V-I trajectory has been conspired using the normalized current and voltage values. The V-I trajectory method divides the class of appliances into eight categories, providing further sub-division within each one. It has been shown that the V-I based method is more accurate than existing methods based on power measurements, for building a taxonomy of electrical appliances due to their sharp V-I curves. 


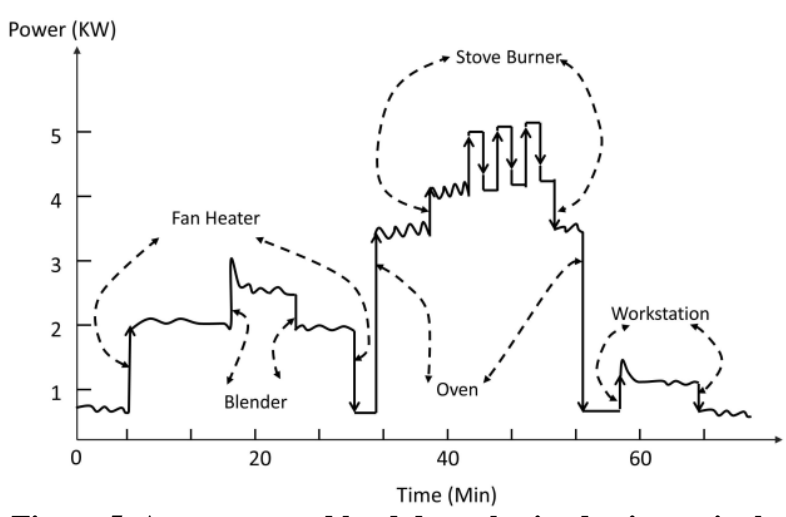

Figure 5. An aggregated load data obtained using a single point of measurement

3.1.1 Loads signatures based on electricity measurement on the fundamental frequency

Another approach to determine consumers is by analyzing their harmonic distortion content. By analyzing the current harmonic spectrum consumers can be classified as linear or non-linear - Figure 6. Starting from this the method can be taken further and based on the present harmonics the devices can be identified.

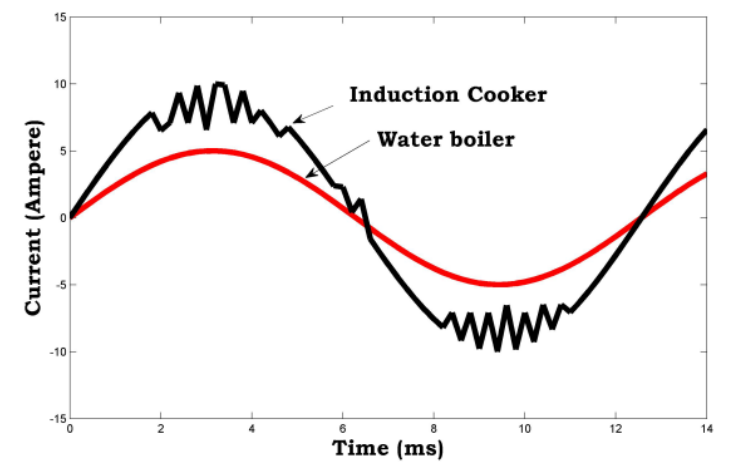

Figure 6. The current draw of linear vs. non-linear loads [19].

It is possible to obtain a recognition equipment with a rich spectrum of harmonics by ratio of all the harmonics with the fundamental one - Figure 7. But for highly resistive loads, it is difficult to make an identification based on the spectrum of harmonics because they have a low level.

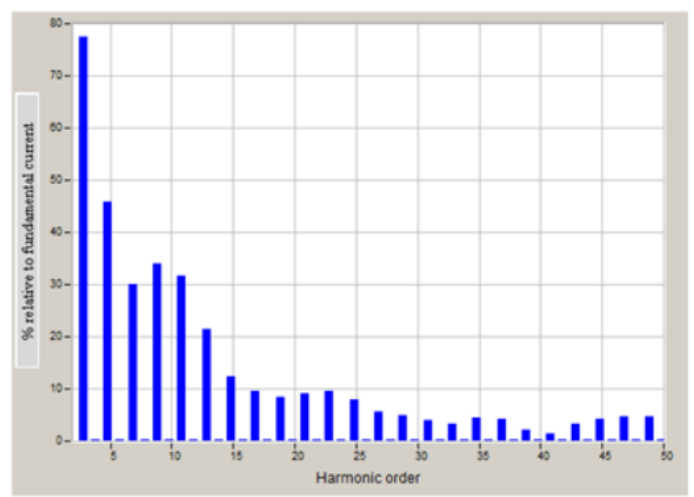

Figure 7. Harmonic up to 50th order (electronic appliance) [34]

\subsection{Loads signatures based on the transient detection}

The primary electrical devices can be recognized from load signatures obtained from transient modes. The transient behaviour of an electrical device (during start-up and shut-off) is a good indicator for identifying the load. The characteristics of the transient load signatures are variable (time, shape, size) because transient measurements depend on the exact value of the voltage at the start/stop time - Figure 8 [34], [23]. Transient displays are used to identify electrical devices which have similar signatures to steady state (but unique transient characteristics when switching between stable states) [31], [35], and [36]. The major disadvantage of the transient analysis is the cost of the equipment used due to their high sampling frequency Also the development of a signature database of unique transient models is another step back.

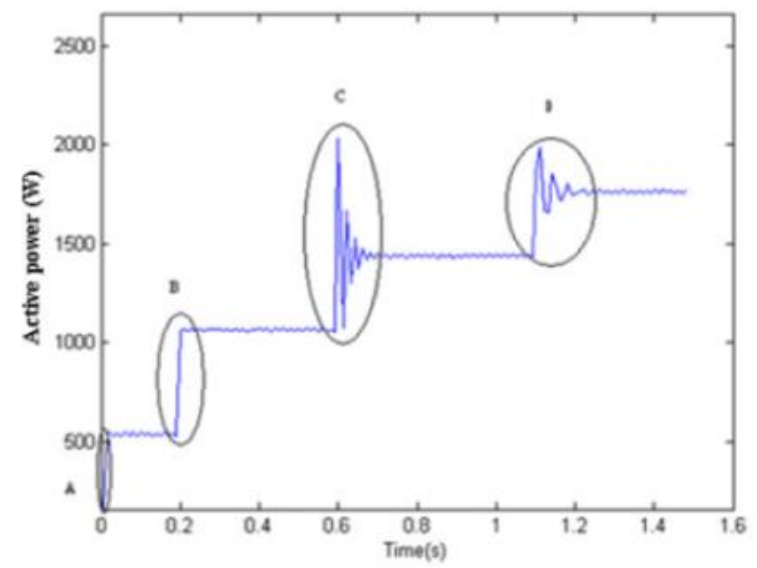

Figure 8. Simultaneous activation of appliances (A, B, C, and D) - transient demarcation on active power consumption [23]

Transient start signal profiles are closely related to the nature of the physical load an electrical appliance needs to perform. For example, consumers using pumps have long-term transitions, while electronic consumers (personal computers, televisions) have short-term transitions of high amplitude. Since the physical tasks to be performed differ from one device to another, the transient start signals can be used for consumer detection. These signals are repetitive and tend to remain in shape even for consumers using power factor correction. A full analysis of the transient profile is difficult to implement and requires considerable resources, which is why an algorithm has been proposed to analyzes only those segments that produce substantial changes, called Vsections. These $\mathrm{V}$-sections are identified by using an average value change detector. The algorithm contains a learning phase in which the characteristic $\mathrm{V}$-sections are identified, followed by the consumer detection stage.

\subsection{Optimal Sampling Frequency for Signature Detection}

The number and kinds of steady-state and transient appliance feature recognizable from an aggregated consumption sample are strongly connected to the 
frequency of measurement in the earliest stages of disaggregation. As noted in [37], a wide range of utilized sampling frequencies is reported in past literature. Many of the steady-state appliance feature described above, such as current harmonics or V-I trajectories and especially transient features are more realistically attainable at higher sampling frequencies. With high frequencies, we usually mean several $\mathrm{kHz}$, although select approaches have even employed $\mathrm{MHz}$ readings [38]. High-frequency sampling rates not only allow more fine-grained and detailed analysis of device-signatures but are also more flexible. The obvious benefit of having more samples available than very few is that when highresolution data is not required or too bulky to store, it can always be down-sampled to lower frequencies. On the other side, metering hardware for high sampling rates is practically non-existent in households today, making NILM techniques requiring sampling rates in the region of $1 \mathrm{~Hz}$ more practical and immediately applicable. $1 \mathrm{~Hz}$ readings as used by Hart's algorithm [15] which has reasonably effectiveness in the examination of active and reactive power measurement. More recently attempts have been made to improve NILM algorithms for the low-frequency sampling of conventional smart meters [39]. For example, in [40] and [41] are developed a method to perform load disaggregation using discriminative sparse coding techniques on power samples provided only on an hourly basis.

\section{CONCLUSIONS}

Current power consumption monitoring solutions are based on the use of smart meters in order to know and control the individual consumption in smart buildings. The implemented methods are grouped into two main categories: intrusive and non-intrusive in the respective networks, each having specific advantages and disadvantages. The paper presents an assessment of the load monitoring solutions based on the analysis of the current situation in the field. Specific hardware / software elements that bring the exactness of each solution, as well as the economic aspects of physical implementation, are indicated.

\section{ACKNOWLEDGEMENTS}

This work was supported by the Grants of the Romanian National Authority for Scientific Research- UEFISCDI, PN-III-P2-2.1-PTE-2016-0024 and PN-III-P2-2.1-BG2016-0346.

\section{REFERENCES}

[1] C. Fischer.Feedback on household electricity consumption: a tool for saving energy. Energy Effic: vol. 1, no. 1, pp. 79-104, 2008.

[2] S. Barker, S. Kalra, and D. Irwin. PowerPlay: Creating Virtual Power Meters through Online Load Tracking. 1st ACM Int. Conf. Embed. Syst. EnergyEfficient Build., pp. 60-69, 2014.

[3] M. Temneanu and A.S. Ardeleanu. Hardware and software architecture of a smart meter based on electrical signature analysis, 8th International Symposium on Advanced Topics in Electrical Engineering (ATEE), pp.1-6, 2013.

[4] M. Temneanu and A. Ardeleanu. Non-intrusive Hybrid Energy Monitoring System. Advanced Materials Research, vol. 837, pp. 495-499, 2014.

[5] G. Seritan, I.Triştiu, O.Ceaki and T.Boboc. Power quality assessment for microgrid scenarios. Electrical and Power Engineering (EPE), 2016 International Conference and Exposition, pp. 723727, 2016.

[6] S. Darby. The Effectiveness of Feedback on Energy Consumption a Review for Defra of the Literature on Metering, Billing. Environ. Chang. Inst. Univ. Oxford, vol. 22, no. April, pp. 1-21, 2006.

[7] A. K. Ehrhardt-Martinez and K. Donnelly. Advanced Metering Initiatives and Residential Feedback Programs : A Meta-Review for Household Electricity-Saving Opportunities. Energy, vol. 123, no. 6, p. 128, 2010.

[8] K. D. Lee, S. B. Leeb, L. K. Norford, P. R. Armstrong, J. Holloway, and S. R. Shaw. Estimation of variable-speed-drive power consumption from harmonic content. IEEE Trans. Energy Convers., vol. 20, no. 3, pp. 566-574, 2005.

[9] W. Wichakool, A. T. Avestruz, R. W. Cox, and S. B. Leeb. Modelling and estimating current harmonics of variable electronic loads. IEEE Trans. Power Electron., vol. 24, no. 12, pp. 2803-2811, 2009.

[10] Z. Wang and G. Zheng. Residential appliances identification and monitoring by a nonintrusive method. IEEE Trans. Smart Grid, vol. 3, no. 1, pp. 80-92, 2012.

[11] Y. K. Cho and M. Gai. Projection-RecognitionProjection Method for Automatic Object Recognition and Registration for Dynamic Heavy Equipment Operations. J. Comput. Civ. Eng., vol. 28, no. 5, pp. 1-9, 2013.

[12] A. Zoha, A. Gluhak, M. A. Imran, and S. Rajasegarar. Non-Intrusive Load Monitoring Approaches for Disaggregated Energy Sensing: A Survey. no. 11, pp. 16838-16866, 2012.

[13] M. Zeifman and K. Roth. Nonintrusive appliance load monitoring: Review and outlook. IEEE Trans. Consum. Electron., vol. 57, no. 1, pp. 76-84, 2011.

[14] M. Baranski and J. Voss. Nonintrusive appliance load monitoring based on an optical sensor. in 2003 IEEE Bologna Power Tech Conference Proceedings, 2003, vol. 4, p. 8 pp. Vol.4.

[15] G. W. Hart. Nonintrusive appliance load monitoring, Proc. IEEE, vol. 80, no. 12, pp. 18701891, 1992.

[16] C. Klemenjak and P. Goldsborough. Non-Intrusive Load Monitoring: A Review and Outlook. 2016.

[17] W. K. Lee, G. S. K. Fung, H. Y. Lam, F. H. Y. Chan, and M. Lucente. Exploration on Load Signatures. Electr. Eng., no. 725, p. 5, 2004.

[18] F. Sultanem. Using appliance signatures for monitoring residential loads at meter panel level. 
IEEE Trans. Power Deliv., vol. 6, no. 4, pp. 13801385, 1991.

[19] J. Liang, S. K. K. Ng, G. Kendall, and J. W. M. Cheng. Load Signature Study - Part I: Basic Concept, Structure, and Methodology. vol. 25, no. 2, pp. 551-560, 2010.

[20] P. Electronics and S. Conference. A Taxonomy of Load Signatures for Single-Phase Electric Appliances. System, vol. 1, no. July 2004, pp. 2429, 2005.

[21] H. Lam, K. Ting, and W. Lee. Building a vectorbased load taxonomy using electrical load signatures. Conf. Electr., no. June, 2005.

[22] H. Lam, G. Fung, and W. Lee. A Novel Method to Construct Taxonomy Electrical Appliances Based on Load Signatures. IEEE Trans. Consum. Electron., vol. 53, no. 2, pp. 653-660, 2007.

[23] H. Najmeddine et al. State of art on load monitoring methods, Proc. 2nd IEEE Int. Conf. Power Energy (PECon 2008), no. PECon 08, pp. 1256-1258, 2008.

[24] J. Paris. A Framework for Non-Intrusive Load Monitoring and Diagnostics. Archives, p. 260, 2006.

[25] M. Baranski and J. Voss. Detecting patterns of appliances from total load data using a dynamic programming approach, Proc. - Fourth IEEE Int. Conf. Data Mining, ICDM 2004, pp. 327-330, 2004.

[26] A. Schoofs, A. Guerrieri, D. T. Delaney, G. M. P. O'Hare, and A. G. Ruzzelli. ANNOT: Automated Electricity Data Annotation Using Wireless Sensor Networks, in 2010 7th Annual IEEE Communications Society Conference on Sensor, Mesh and Ad Hoc Communications and Networks (SECON), 2010, pp. 1-9.

[27] S. Park, H. Kim, H. Moon, J. Heo, and S. Yoon. Concurrent simulation platform for energy-aware smart metering systems. IEEE Trans. Consum. Electron., vol. 56, no. 3, pp. 1918-1926, 2010.

[28] T. Kato, H. S. Cho, D. Lee, T. Toyomura, and T. Yamazaki. Appliance recognition from electric current signals for information-energy integrated network in home environments. Lect. Notes Comput. Sci., vol. 5597 LNCS, pp. 150-157, 2009.

[29] M. E. Berges, E. Goldman, H. S. Matthews, and L. Soibelman. Enhancing electricity audits in residential buildings with nonintrusive load monitoring. J. Ind. Ecol., vol. 14, no. 5, pp. 844$858,2010$.

[30] L. Farinaccio and R. Zmeureanu. Using a pattern recognition approach to disaggregate the total electricity consumption in a house into the major end-uses. Energy Build., vol. 30, no. 3, pp. 245259, Aug. 1999.

[31] M. T. Fitta. Load Classification and Appliance Fingerprinting for Residential Load Monitoring System. English, p. 100, 2010.

[32] C. Cepisca, S. Ganatsios, H.Andrei, S.D. Grigorescu, and N. Taousanidis, Methods for power measurements in energy meters, The Scientific
Bulletin of Electrical Engineering Faculty, 2008, year 8, no. 2, pp. 7-13.

[33] C. Laughman et al. Power signature analysis. IEEE Power Energy Mag., vol. 1, no. 2, pp. 56-63, 2003.

[34] M. Berges, E. Goldman, H. S. Matthews, and L. Soibelman. Training Load Monitoring Algorithms on Highly Sub-Metered Home Electricity Consumption Data. Tsinghua Sci. Technol., vol. 13, no. SUPPL. 1, pp. 406-411, 2008.

[35] M. Hazas, A. Friday, and J. Scott. Look back before leaping forward: Four decades of domestic energy inquiry. IEEE Pervasive Comput., vol. 10, no. 1, pp. 13-19, 2011.

[36] D. D. Sabin, D. L. Brooks, and A. Sundaram. Indices for assessing harmonic distortion from power quality measurements: definitions and benchmark data. IEEE Trans. Power Deliv., vol. 14, no. 2, pp. 489-496, 1999.

[37] D. Srinivasan, W. S. Ng, and A. C. Liew. Neuralnetwork-based signature recognition for harmonic source identification. IEEE Trans. Power Deliv., vol. 21, no. 1, pp. 398-405, 2006.

[38] J. Z. Kolter and M. J. Johnson. REDD : A Public Data Set for Energy Disaggregation Research. SustKDD Work., vol. xxxxx, no. 1, pp. 1-6, 2011.

[39] S. Gupta, M. S. Reynolds, and S. N. Patel. ElectriSense: single-point sensing using EMI for electrical event detection and classification in the home. Proc. 12th ACM Int. Conf. Ubiquitous Comput., pp. 139-148, 2010.

[40] J. Z. Kolter, S. Batra, and A. Y. Ng. Energy disaggregation via discriminative sparse coding. Adv. Neural Inf. Process. Syst., pp. 1153-1161, 2010.

[41] O.M. Ghita, S.D. Grigorescu, H. Andrei, and N. Calin, Solution for inspection of Power Energy Equipment Using Augmented Reality, The Scientific Bulletin of Electrical Engineering Faculty, 2017, no. 1, https://doi.org/10.1515/sbeef2016-0007. 